Pneumologe $2005 \cdot 2: 201-204$ DOI 10.1007/s10405-005-0042-3

Online publiziert: 26. April 2005

๑) Springer Medizin Verlag 2005

\section{Redaktion}

F. J. Meyer, Heidelberg

T. E. Wessendorf, Essen
B. Hoksch - T. Weber · M. Beshay - R. Stein · J. Schardt · R. A. Schmid

DMLL - Klinik für Thoraxchirurgie, Universitätsspital Bern

\title{
Pulmonales Infiltrat nach Pneumothoraxdrainage
}

\section{Anamnese}

Die 46-jährige Patientin stellte sich in unserer Notfallambulanz mit seit 1 Woche bestehendem Hustenreiz und Dyspnoe vor. Keine Haemoptoe, kein Hautemphysem.

\section{Befunde}

Fünf Tage zuvor war vom Hausarzt eine Thoraxröntgenaufnahme vorgenommen worden, die einen Pneumothorax rechts von ca. $4 \mathrm{~cm}$ Breite nachwies. Eine Drainageneinlage oder Punktion des Pneumothorax war zum damaligen Zeitpunkt nicht er- folgt. In der aktuellen Röntgenaufnahme sah man jetzt einen Totalkollaps der rechten Lunge ohne Mediastinalverschiebung.

\section{Therapie}

Unmittelbar nach Einlage einer Thoraxdrainage in Bülau-Technik und Anlage von $-20 \mathrm{~cm} \mathrm{H}_{2} \mathrm{O}$-Sog klagte die Patientin über eine Zunahme des Hustenreizes mit teilweise schaumigem Auswurf. Darüber hinaus entwickelte die Patientin eine leichte Hypoxämie. Zur weiteren Abklärung wurde eine Computertomographie durchgeführt (• Abb. 1a, b).
Hier kann auch Ihr Fall dargestellt werden!

Haben Sie Anregungen oder eine interessante Falldarstellung?

Senden Sie diese bitte an:

Dr. F. Joachim Meyer Medizinische Klinik und Poliklinik Abt. Innere Medizin III Im Neuenheimer Feld 410 69120 Heidelberg Joachim.Meyer@ med.uni-heidelberg.de

Dr. Thomas E. Wessendorf Ruhrlandklinik Tüschener Weg 40 45239 Essen thomas.wessendorf@ ruhrlandklinik.de
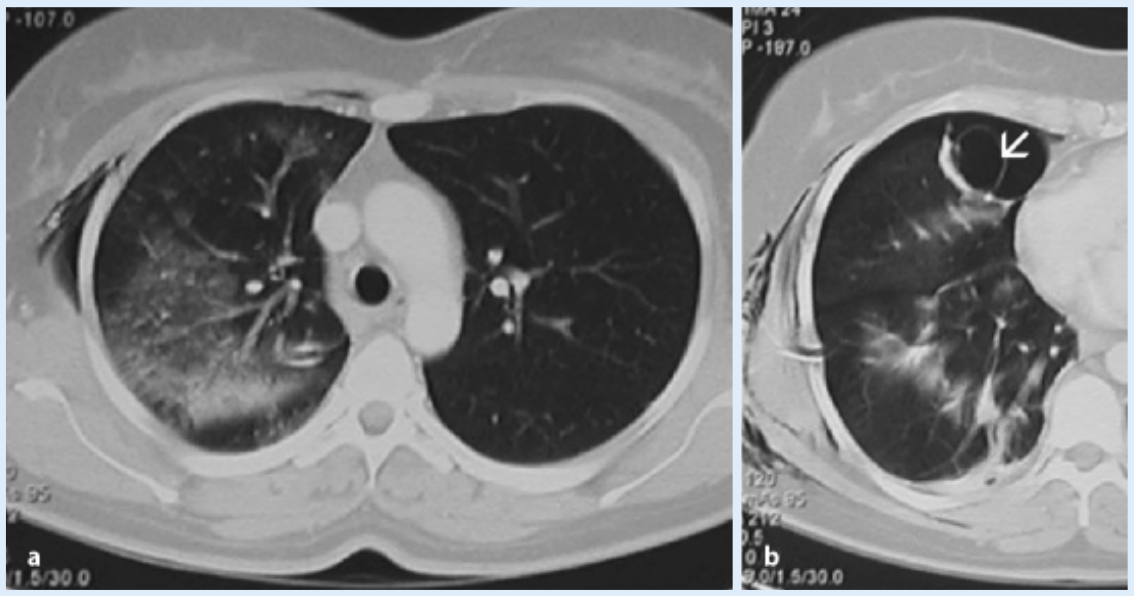

Abb. 1a, b $\triangle$ Computertomographie der Lunge, Bulla im ML (Pfeil), leichtes Hautemphysem nach Draineinlage 
Hier steht eine Anzeige.

黛 Springer 


\section{\ Diagnose: Reexpansionsödem der Lunge}

Bei afebriler und laborchemisch (Leukozyten, CrP) unauffälliger Konstellation ohne anamnestische Hinweise auf stattgehabte oder aktuelle Infektion der Luftwege musste die primäre Verdachtsdiagnose einer Pneumonie verlassen werden. Das breitflächige Infiltrat $(\bullet$ Abb. 1a) wurde dann als ein Reexpansionsödem identifiziert.

\section{Verlauf}

Da die Patientin keine weiteren schwerwiegenden respiratorischen Einschränkungen entwickelte, konnte auf eine Respiratortherapie verzichtet und eine symptomatische Therapie eingeleitet werden. Das KontrollCT 48 h später wies eine Regredienz des Ödems nach (• Abb. 2). Als Ursache des Pneumothorax konnte im CT eine bullöse Veränderung im Mittellappen ausgemacht werden, die nach Stabilisierung des Allgemeinzustandes der Patientin thorakoskopisch reseziert wurde; außerdem wurde eine Pleuraabrasio durchgeführt (- Abb. 3). Die Patientin verließ bei weiter unauffälligem Verlauf am 6. postoperativen Tag die Klinik.

\section{Diskussion}

Das Reexpansionsödem, 1958 erstmalig von Carlson beschrieben, tritt vor allem bei einem „chronischen“, ausgedehnten Pneumothorax oder Pleuraerguss auf, wird aber auch bei einem kleinen Pneumothorax, der erst kurzzeitig besteht, beschrieben. Critchley et al. berichten weiterhin über ein Reexpansionsödem im Rahmen der thorakoskopischen Evakuation eines Pleuraergusses, Scalzetti et al. beobachteten diese Komplikation nach Talkpleurodese $[2,6,7]$. Ein Reexpansionsödem nach Highfrequency-Jet-Ventilation bei einer Patientin mit VATS bei Spontanpneumothorax wird von Fujino et al. beschrieben [3].

\section{Pathogenese}

Zur Pathogenese des Reexpansionsödems werden parallel zu Untersuchungen beim ARDS, Postpneumonektomieödem und Lungentransplantation $u$. a. eine gesteigerte vaskuläre Permeabilität der hypoxisch geschädigten Lunge und ein SurfactantVerlust diskutiert $[8,9]$.
In tierexperimentellen Studien wurde die Rolle von biochemischen Mediatoren untersucht. Dabei konnte gezeigt werden, dass insbesondere IL8 und MCP1 eine entscheidende Rolle bei der Entwicklung des Reexpansionsödems zu spielen scheinen [5]. Im Jahr 2002 gelang Suzuki et al. der Zytokinnachweis in der Ödemflüssigkeit der Lunge [9]. Weiterhin steigern aktivierte freie Radikale die mikrovaskuläre Permeabilität im Rahmen der Lipidperoxidationskaskade [8].

\section{Klinik und Therapie}

Die Klinik des Reexpansionsödems kann vom asymptomatischen Zufallsbefund bis hin zu schwerwiegenden kardiorespiratorischen Einschränkungen mit der Notwendigkeit zur kurzzeitigen Respiratortherapie reichen. Entsprechend ist die Therapie dem klinischen Bild anzupassen, wobei eine CPAP-Beatmung bei respiratorischer Insuffizienz favorisiert wird [4]. Die Symptomatik (Husten, schaumiger Auswurf, Dyspnoe bis hin zur Ateminsuffizienz) kann dabei entweder sofort, d. h. unmittelbar nach der Reexpansion der Lunge, oder mit einer Verzögerung von bis zu 24 h einsetzen. In der Regel sind die Befunde - wie auch im Fall unserer Patientin - innerhalb von 48-72 h regredient. Klinisch sowie radiologisch lässt sich ein akuter Pneumothorax im Sinne eines

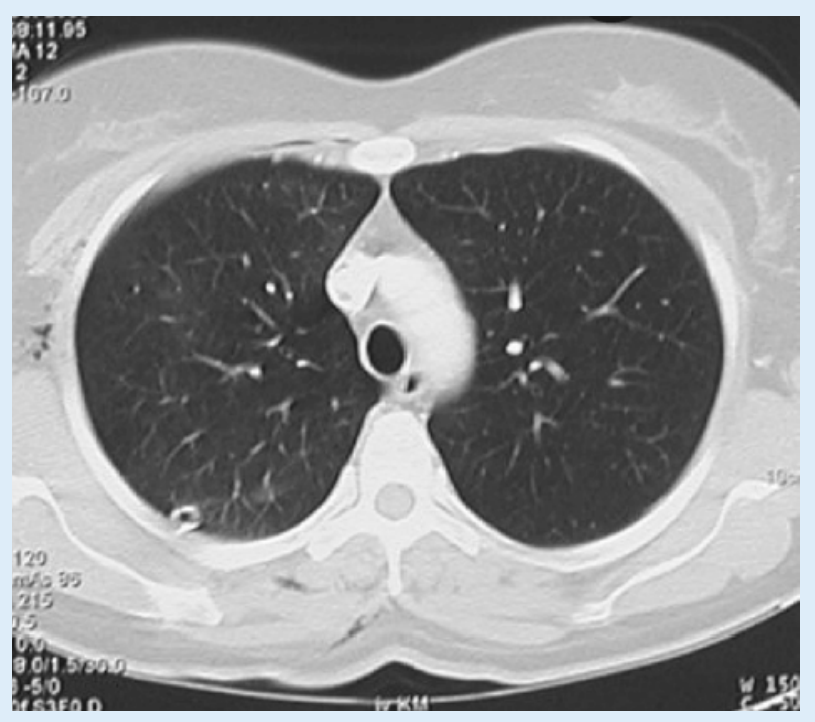

Abb. $2 \triangle$ Deutliche Regredienz des Ödems im Kontroll-CT

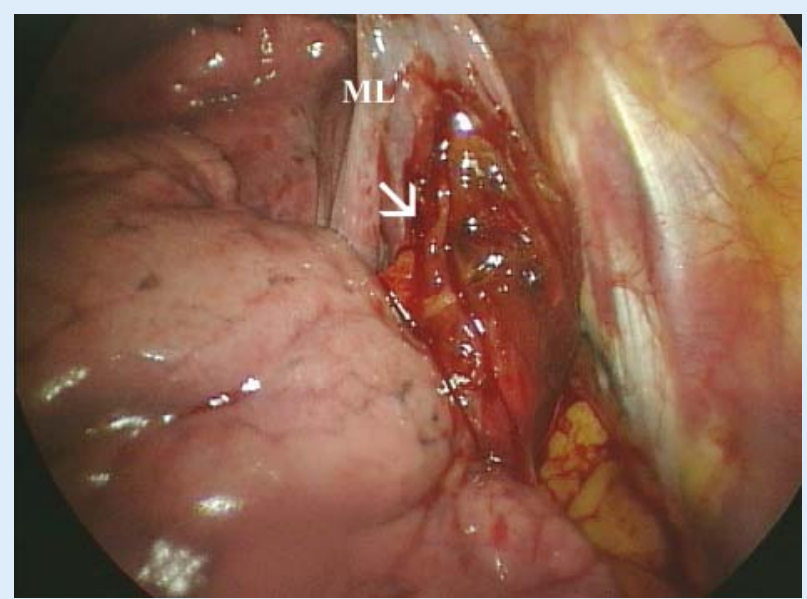

Abb. $3 \Delta$ Exposition der zu resezierenden eingebluteten Bulla (Pfeil) im Mittellappen (ML) 
Spannungspneumothorax sicher abgrenzen. Die ödematösen Veränderungen, die oft fälschlicherweise als entzündliche Infiltrate fehlgedeutet werden, treten in der Regel ipsilateral auf. In einer Sammelstatistik von Mahfood et al. wird die Gesamtmortalitätsrate beim Reexpansionsödem mit 19\% (9 von insgesamt 47 Patienten) angegeben [4]. Patienten mit einem Reexpansionsödem sollten einer entsprechenden kardiopulmonalen Überwachung zugeführt werden.

- Generell, aber insbesondere bei einem länger bestehenden Pneumothorax oder einem ausgedehnten Pleuraerguss, muss mit der Entstehung eines Reexpansionsödems - auch bei langsamer Reexpansion der Lunge gerechnet werden.

In Anlehnung an die Empfehlungen des American College of Chest Physicians (ACCP) von 2001 sollte daher bei einem ausgedehnten Pneumothorax $(\geq 30 \%$ der Lunge) auf einen unmittelbaren Anschluss der Thoraxdrainage an einen Sog verzichtet werden [1]. Ob diese Technik im vorliegenden Fall die Ausbildung eines Reexpansionsödems verhindert hätte, bleibt allerdings ungewiss.

\section{Korrespondierender Autor \\ Dr. B. Hoksch}

DMLL - Klinik für Thoraxchirurgie, Universitätsspital, 3010 Bern, Schweiz

E-Mail: beatrix.hoksch@insel.ch

Dieser Beitrag ist in leicht modifizierter Form in Der Chirurg (2005) 76:270-272 erschienen.

Interessenkonflikt: Der korrespondierende Autor versichert, dass keine Verbindungen mit einer Firma, deren Produkt in dem Artikel genannt ist, oder einer Firma, die ein Konkurrenzprodukt vertreibt, bestehen.

\section{Literatur}

1. Baumann MH, Strange C, Heffner JE et al. (2001) Management of spontaneous pneumothorax - An American College of Chest Physicians Delphi Consensus Statement. Chest 119:590-602

2. Critchley LA, Au HK, Yim APC (1996) Reexpansion pulmonary edema occuring after thoracoscopic drainage of a pleural effusion. J Clin Anesth 8:591594
3. Fujino S, Tezuka N, Inoue S et al. (2000) Reexpansion pulmonary edema due to highfrequency jet ventilation: report of a case. Surg Today 30:1110 1111

4. Mahfood S, Hix WR, Aaron BL et al. (1988) Reexpansion pulmonary edema. Ann Thorac Surg 45:340 345

5. Sakao Y, Kajikawa O, Martin TR et al. (2001) Association of IL8 and MCP1 with the development of reexpansion pulmonary edema in rabbits. Ann Thorac Surg 71:1825-1832

6. Scalzetti EM (2001) Unilateral pulmonary edema after talc pleurodesis. J Thorac Imag 16:99-102

7. Sherman SC (2003) Reexpansion pulmonary edema: a case report and review of the current literature. J Emerg Med 24:23-27

8. Sivrikoz MC, Tuncözgür B, Cekmen M et al. (2002) The role of tissue reperfusion in the reexppansion injury of the lungs. Eur J Cardio Thorac Surg 22:721-727

9. Suzuki S, Niikawa H, Shibuya J et al. (2002) Analysis of edema fluids and histologic features of the lung in reexpansion pulmonary edema during videoassisted thoracoscopic surgery. J Thorac Cardiovasc Surg 123:387-389

\section{Patientensicherheit}

Eine Kernaufgabe des ÄZQ

Themen wie Patientensicherheit und Fehlermanagement gehörten in der Vergangenheit eher zu den wenig spektakulären in der Gesundheitsversorgung. Dennoch erklärte sie das Ärztliche Zentrum für Qualität in der Medizin anlässlich seines 10jährigen Bestehens zu den Schwerpunkten seiner künftigen Arbeit. Der Grund hierfür ist die immer weiter zunehmende Leistungsfähigkeit der Medizin einerseits und die Zunahme an älteren und multimorbiden Patienten andererseits. Eine hohe Leistungsfähigkeit ist jedoch nicht mit Fehlerfreiheit oder gar Unfehlbarkeit gleichzusetzen. Gerade die Möglichkeiten, die die moderne Medizin bietet, bergen auch Fehlerpotenziale in sich. Ein Fehler aber ist dann keine Katastrophe, wenn frühzeitig eine kritische Auseinandersetzung mit der Fehlervermeidung bzw. des Umganges mit Fehlern erfolgt.

Vor diesem Hintergrund sieht es das ÄZQ als außerordentlich wichtig an, Fragen zur Patientensicherheit und zum kritischen und konstruktiven Umgange mit Fehlern zum Inhalt seiner Arbeit zu machen. Ein erster Schritt in diese Richtung ist der 2005 erschienene "Leitfaden Patientensicherheit" (Holzer E, Thomeczek C, Hauke E, Conen $D$, Hochreutnener M-A, editors. Patientensicherheit - Leitfaden für den Umgang mit Risiken im Gesundheitswesen. Wien: Facultas; 2005. ISBN 3-85076-687-X.).

In Ergänzung dieses Leitfadens wurde das themenspezifische Internetportal http://www.forum-patientensicherheit. de/ eingerichtet. In diesem Portal wird das ÄZQ - unterstützt durch einen Redaktionsbeirat - ab jetzt aktuell über die Themen Fehlermanagement und Patientensicherheit berichten. Hierzu gehören theoretische Aspekte und die Information über verschiedene deutsche und internationale Programme. In einer Sammlung weiterführender Literatur findet der Leser einen Überblick über die Standardwerke zum Thema.

Quelle: Ärztliches Zentrum für Qualität in der Medizin, Berlin, Internet: www.azq.de Email:Saenger@azq.de 